\title{
Towards SU(2) invariant formulation of the monopole confinement mechanism
}

\author{
Katsuya Ishiguro*i \\ Institute for Theoretical Physics, Kanazawa University, Kanazawa 920-1192, Japan \\ and RIKEN, Radiation Laboratory, Wako 351-0158, Japan \\ E-mail: ishiguro@hep.s.kanazawa-u.ac.jp
}

\section{Yoshihiro Mori, Yoshifumi Nakamura, Toru Sekido and Tsuneo Suzuki ${ }^{+}$ \\ Institute for Theoretical Physics, Kanazawa University, Kanazawa 920-1192, Japan \\ and RIKEN, Radiation Laboratory, Wako 351-0158, Japan}

M. N. Chernodub and M. I. Polikarpov ${ }^{\S}$

Institute of Theoretical and Experimental Physics ITEP, 117259 Moscow, Russia

\section{I. Zakharov}

Max-Planck Institut für Physik, Föhringer Ring 6, 80805, München, Germany

\begin{abstract}
The type of the vacuum is studied numerically in the maximally Abelian (MA) gauge and in the Landau (LA) gauge of $S U(2)$ gluodynamics. The type of the vacuum is determined by a ratio between the dual coherence and the dual penetration lengths. The dual penetration length is determined from correlations between Wilson loops and electric fields in both gauges. The dual coherence length is found from correlations between Wilson loops and dimension-2 operators both in the MA and the LA gauges. This determination of the coherence length is supported by theoretical and numerical observation that the dimension-2 gluon operators in the studied gauges have a strong correlation with the monopole current determined in the MA gauge. We find numerically that the dual penetration lengths and the dual coherence lengths in the LA and the MA gauges are almost the same. Therefore we conclude, that in both gauges the type of the vacuum in the confinement phase is near to the border between the type 1 and the type 2 dual superconductors.
\end{abstract}

XXIIIrd International Symposium on Lattice Field Theory

25-30 July 2005

Trinity College, Dublin, Ireland

\footnotetext{
* Speaker.

${ }^{\dagger}$ The authors thank RIKEN for their support of computer facilities and use of the RSCC computer clusters.

†.S. is supported by JSPS Grant-in-Aid for Scientific Research on Priority Areas 13135210 and (B) 15340073.

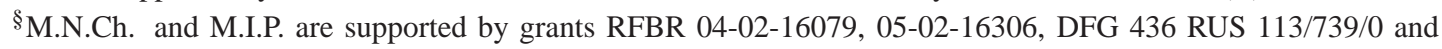
MK-4019.2004.2.
} 


\section{Introduction}

It is conjectured that the dual Meissner effect caused by the monopole condensation is the color confinement mechanism [1]. The conjecture seems to be realized if we perform Abelian projection in the maximally Abelian (MA) gauge where Abelian component of the gluon field and Abelian monopoles are found to be dominant [2]. Numerical calculations show that the vacuum of quenched $S U(2)$ QCD ( $S U(2)$ gluodynamics) is near the border between the type 1 and the type 2 dual superconductor [3], although there are some claims that it is a superconductor of weakly type 1, Refs. [4, 5]. Since the explicit definition of a dual Higgs field is unknown the coherence length $\xi$ is usually calculated using classical Ginzburg-Landau equations, while the penetration length $\lambda$ can be calculated directly measuring the correlations between Wilson loops and (non-)Abelian electric fields. Below we show that the coherence length $\xi$ can be derived in the MA gauge also from the measurement of the monopole density around a chromomagnetic flux.

The MA gauge is just one gauge among infinite possible gauges. Since the physics should be gauge-independent, it is important to know the confinement mechanism as well as the type of the vacuum in another gauge. This problem has been discussed recently in Ref. [6] where the Landau (LA) gauge is considered and for Abelian components the dual Meissner effect is observed. A magnetic displacement current plays the role of the solenoidal supercurrent which squeezes the Abelian electric fields. The observation of the dual Meissner effect in the LA gauge suggests that there exists a gauge-independent definition of the monopole condensation.

In order to fix the type of the vacuum in the MA and in the LA gauges we use the following scheme. First, we demonstrate numerically a strong correlation between the dimension 2 gluon operators (namely, the operator $A^{+} A^{-}(s) \equiv \sum_{\mu}\left[\left(A_{\mu}^{1}(s)\right)^{2}+\left(A_{\mu}^{2}(s)\right)^{2}\right]$ in the MA gauge and $A^{2}(s)=$ $A^{+} A^{-}(s)+A^{3} A^{3}(s)$ in the LA gauge [7]) and the monopole currents $\left|k_{\mu}(s)\right|$ (defined in the MA gauge). Then we show that the monopole density is strongly correlated with the position of the QCD string. Indeed, in the dual Ginzburg-Landau model [8] of the SU(2) vacuum, the expectation value of the (squared) monopole density around the string worldsheet $\Sigma$ is given by the sum of the solenoidal current and the quantum correction, respectively [9]:

$$
\begin{aligned}
\left\langle k_{\mu}^{2}\right\rangle_{\Sigma} \equiv & \left(k_{\mu}^{\text {string }}\right)^{2}+\left(k_{\mu}^{\text {quant }}\right)^{2}=\left[\eta^{2} m_{B} K_{1}\left(m_{B} \rho\right)\right]^{2}+\frac{g^{2}|\Phi(\rho)|^{4} \Lambda^{2}}{16 \pi^{2}}+\ldots \\
& \left.\rightarrow \frac{g^{2} \Lambda^{2} \eta^{4}}{16 \pi^{2}}\left[1-4 \sqrt{\frac{\pi \xi}{2 \rho}} e^{-\rho / \xi}\right]+\ldots \quad \text { [in the limit } \rho \gg \xi\right] .
\end{aligned}
$$

Here $m_{B} \equiv 1 / \lambda$ is the mass of the dual gauge boson, $\eta$ is the expectation value of the Higgs field, $\Lambda$ is an UV-cutoff, and $\rho$ is the distance to the string worldsheet (the string is taken to be infinitely long, straight and static for the sake of simplicity). Moreover, the limit (1.1) demonstrates that the leading behavior of the monopole density at large distances is controlled by the coherence length $\xi$ and not by the penetration length $\lambda$.

Thus, coherence lengthes in the MA and LA gauges can be found from the correlation between the corresponding dimension 2 operators and the Wilson loops. The comparison of the penetration length and the coherence lengthes reveals that they are almost the same. Consequently, we conclude that the vacuum is near the border between the type 1 and the type 2 dual superconductors in the MA gauge. Below we show the results of the numerical simulations which support our conclusion. 


\section{Numerical results}

\subsection{Method}

We use an improved gluonic action found by Iwasaki [10] which was already implemented in Ref. [6]: $S=\beta\left\{C_{0} \sum \operatorname{Tr}\right.$ (plaquette) $+C_{1} \sum \operatorname{Tr}$ (rectangular) $\}$. The mixing parameters are fixed as $C_{0}+8 C_{1}=1$ and $C_{1}=-0.331$. We adopt the coupling constant $\beta=1.2$ which corresponds to the lattice spacing $a(\beta=1.2)=0.0792(2) \mathrm{fm}$. The lattice size is $32^{4}$ and we use around 5000 thermalized configurations for measurements. To get a good signal-to-noise ratio, the APE smearing technique [11] is used when evaluating Wilson loops $W(R, T)=W^{0}+i W^{a} \sigma^{a}$. The thermalized vacuum configurations are gauge-transformed in the MA(+ U1LA) gauge and in the LA gauge.

\subsection{MA gauge}

The MA gauge is defined by the maximization of the functional $R[U]=\sum_{l} R_{l}[U]$, where $R_{l}[U]=\frac{1}{2} \operatorname{Tr}\left[U_{l} \sigma_{3} U_{l}^{\dagger} \sigma_{3}\right]$, with respect to the $\mathrm{SU}(2)$ gauge transformations, $U_{x, \mu}^{\Omega}=\Omega_{x}^{\dagger} U_{x, \mu} \Omega_{x+\mu}$. In a naive continuum limit one can make an identification of the dimension-2 operator $A_{\mu}^{+} A_{\mu}^{-}$and a lattice quantity: $A_{\mu}^{+}(x) A_{\mu}^{-}(x)=\frac{1}{2}\left(1-R_{x, \mu}[U]\right)$ where no summation over $\mu$ is assumed.

The numerical measurements [12] of the local correlation between monopoles and the quantity $R_{l}$ revealed that the $A^{+} A^{-}$condensate is enhanced on monopoles. Moreover, according to Fig. 1 the correlation between the $A^{+} A^{-}$condensate and the monopole is short ranged with the correlation length $\zeta_{\text {cond }} \approx 0.06 \mathrm{fm}$. Note that $\zeta_{\text {cond }} \approx \zeta_{\text {Action }} \approx 0.05 \mathrm{fm}$ where the $\zeta_{\text {Action }}$ is the scale of correlations between the monopole density and the $\mathrm{SU}(2)$ action [13].

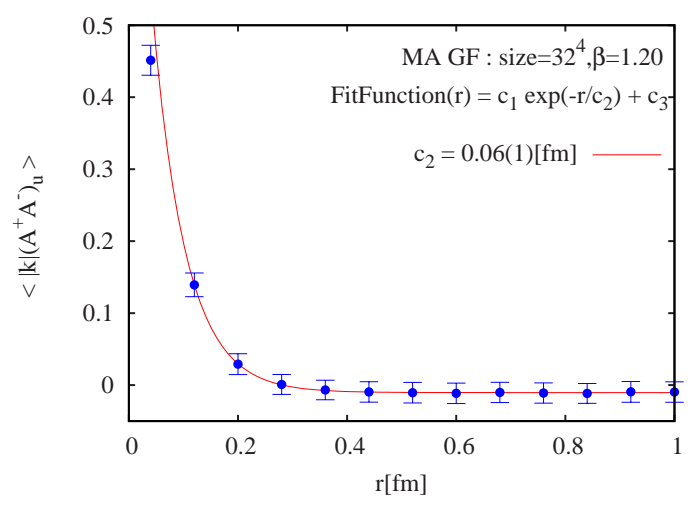

Figure 1: The correlation between the monopole density $\left|k_{\mu}\right|$ and the operator $A^{+} A^{-}$in the MA gauge. The solid line denotes the best exponential fit.

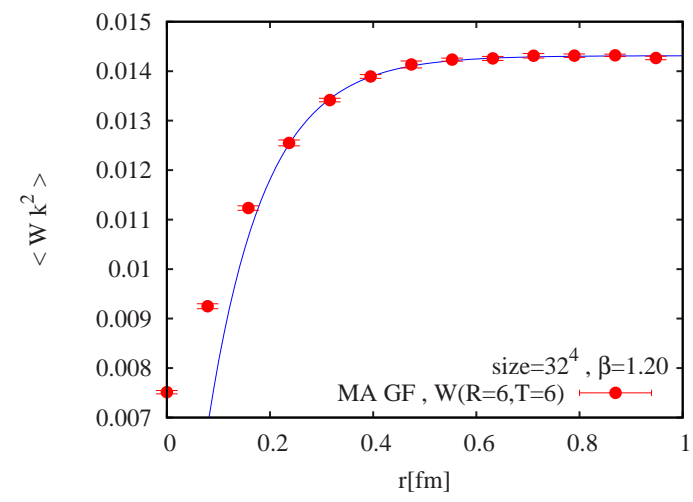

Figure 2: The correlation between the $R \times T=$ $6 \times 6$ Wilson loop and the square monopole density in the MA gauge. The solid line denotes the best exponential fit.

\subsubsection{Correlations of monopoles and condensates with chromoelectric strings}

Let us then derive the coherence length in the MA gauge. The correlations between the Wilson loop and the monopole density is plotted in Fig. 2, The static quarks are represented by the Wilson loop $W(R, T)$. The measurements of correlations are mainly done on the perpendicular plane at 
the midpoint between the quark pair. An exponential fit of the correlation function provides the correlation length of the vacuum $\xi$ according to our discussion above.

Since the monopoles and the dimension-2 condensate are strongly correlated, the coherence length can also be calculated from the correlations between the Wilson loop and the dimension-2 quantity $\left[A^{+} A^{-}(s)\right]_{\theta}=\sum_{\mu}\left\{\left[\theta_{\mu}^{1}(s)\right]^{2}+\left[\theta_{\mu}^{2}(s)\right]^{2}\right\}$ which uses the angles $\theta_{\mu}(s)$ given by the relation $U_{\mu}(s)=\exp \left(i \theta_{\mu}^{a}(s) \sigma^{a}\right)$. The quantity $\left[A^{+} A^{-}(s)\right]_{\theta}$ (measured in the MA+U1LA gauge) is identical in naive continuum limit to the quantity $A^{+} A^{-}(s)$ defined in the MA gauge. The corresponding correlation function is shown in Fig. 3, We find the coherence lengths determined by the use of the quantities $\left[A^{+} A^{-}\right]_{\theta}$ and $k^{2}$ coincide within the error bars.

To derive the penetration length we study the correlation of the non-Abelian electric fields which are defined from $1 \times 1$ plaquette $U_{\mu \nu}(s)=U_{\mu v}^{0}(s)+i U_{\mu v}^{a}(s) \sigma^{a}$. A typical example is shown in Fig. 4. Note that electric fields perpendicular to the $Q \bar{Q}$ axis are found to be negligible. An exponential fit of this correlation function provides the penetration length of the vacuum, $\lambda$.

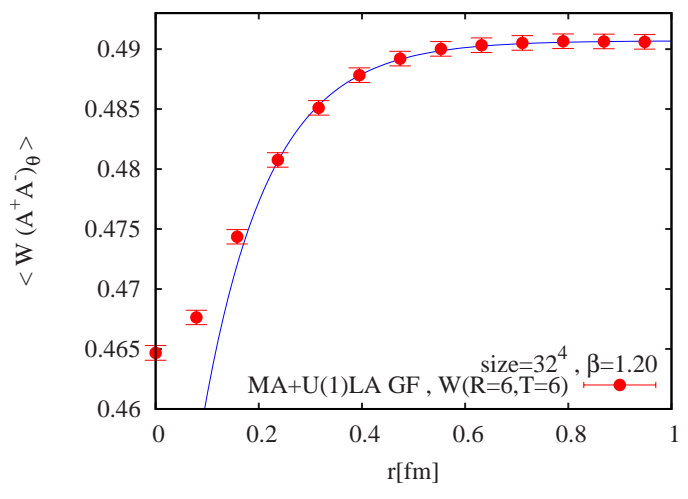

Figure 3: The correlation between the $R \times T=$ $6 \times 6$ Wilson loop and the $A^{+} A_{\theta}^{-}$in the MA + U1LA gauge. The solid line denotes the best exponential fit.

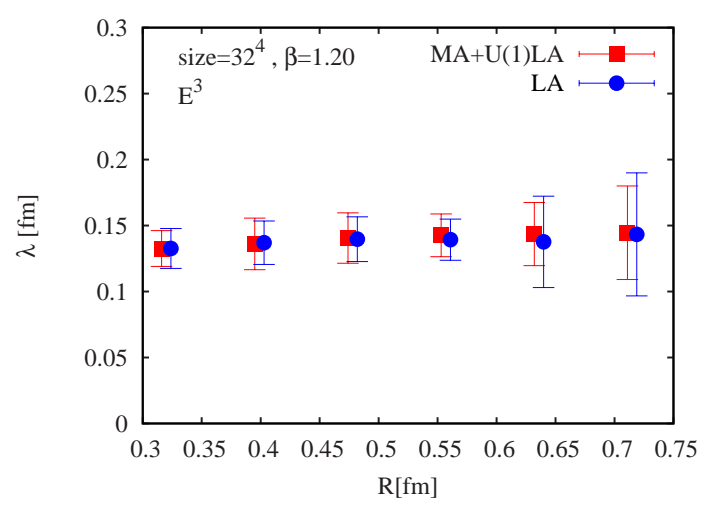

Figure 5: The penetration lengths of the nonAbelian electric field in the Landau gauge and in the MA + U1LA gauge for various $R$.

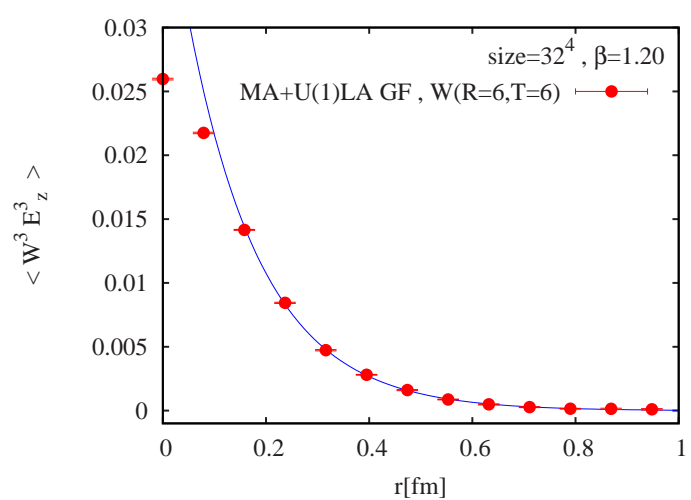

Figure 4: The non-Abelian $\vec{E}$ electric field profile in the MA + U1LA gauge obtained with the use of the $R \times T=6 \times 6$ Wilson loop. The solid line denotes the best exponential fit.

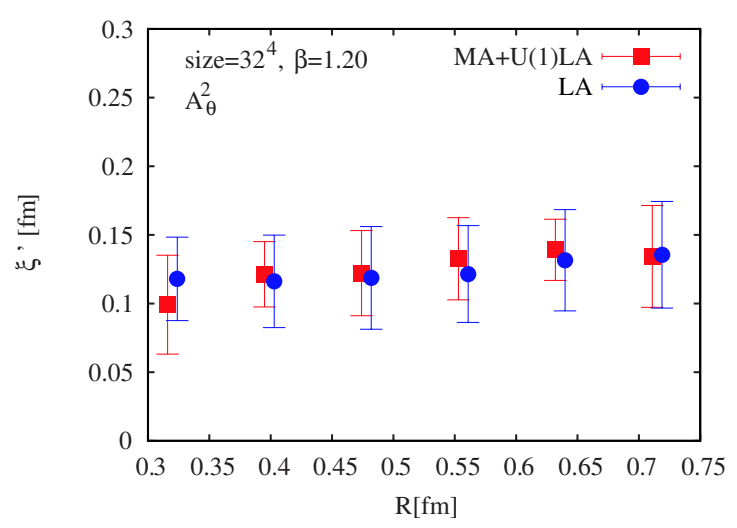

Figure 6: The coherence lengths of the dimension 2 gluon operator in the Landau gauge and in the MA + U1LA gauge for various $R$. 


\subsection{LA gauge}

In the LA gauge the functional $\sum_{s, \mu} \operatorname{Tr}\left[U_{\mu}(s)+U_{\mu}^{\dagger}(s)\right]$ is maximized with respect to all gauge transformations. Similarly to the case of the MA gauge, we find the coherence length from the measurement of the correlation between the chromoelectric string and the dimension- 2 operator

$$
A^{2}(s) \equiv \sum_{\mu=1}^{4} \sum_{a=1}^{3}\left(\theta_{\mu}^{a}(s)\right)^{2} .
$$

Indeed, in the LA gauge the operator $A^{2}(s)$ (or its square-root) is physically relevant and may have information about properties of a dual Higgs field characterizing the QCD vacuum.

The $A^{2}(s)$ profile around the string in the LA gauge is very similar to that shown in Fig. 3 for the MA gauge. This is very exciting, since the behavior of the profile is just what we expect from a profile of a Higgs field. The coherence length is obtained from the exponential fitting of the correlation function similarly to the MA case.

To derive the penetration length we study the correlation of the Wilson loops with electric fields $E_{A i}^{a}$ defined in the LA gauge. The correlations in the LA gauge are very similar to the case of the MA gauge shown in Fig. 4 .

\subsection{The vacuum type: comparison between MA gauge and LA gauge}

In order to study the gauge-(in)dependence of the dual superconductor picture, we show in Fig. 5 the penetration lengths determined in the MA + U1LA gauge and in the LA gauge. We also plot the coherence lengths in Fig. 6. From these figures, we observe that the coherence and correlation lengths calculated in different gauges coincide with each other. Note that we measure the correlation between the gauge-invariant Wilson loop and the gauge-invariant ("gauge-singlet") pieces of the a gauge-variant operators $A^{2}$. Since the $A^{2}$ operators are defined with respect to different gauges their gauge-singlet parts are non-local and different. Therefore the observed equivalence of the correlations lengths is a non-trivial fact.

The Ginzburg-Landau parameter (i.e., the ratio of penetration length and the coherence length) determines the type of the SU(2) vacuum. According to our measurements

$$
\begin{aligned}
\kappa_{M A} & =1.04( \pm 0.07 \text { statistic })( \pm 0.1 \text { systematic }) & & {[\text { MA gauge }] } \\
\kappa_{L A} & =1.04( \pm 0.05 \text { statistic })( \pm 0.1 \text { systematic }) & & {[\text { LA gauge }] }
\end{aligned}
$$

\section{Conclusions}

1. The coherence lengths of the vacuum of the SU(2) gluodynamics in the MA gauge can equivalently be fixed either (i) from the correlations between the Wilson loops and the monopole density, or (ii) from the correlations between the Wilson loops and the dimension 2 operators.

2. The coherence lengths measured in the MA gauge and in the LA gauge are the same.

3. The penetration lengths measured in the MA gauge and in the LA gauge are the same.

4. The type of the vacuum in both gauges is determined to be near the border between type 1 and type 2. The Ginzburg-Landau parameters in both gauges coincide with each other. 


\section{References}

[1] Y. Nambu, Strings, monopoles, and gauge fields, Phys. Rev. D 10 (1974) 4262; G. 't Hooft, in High Energy Physics, ed. A. Zichichi, EPS International Conference, Palermo, 1975; S. Mandelstam, Vortices and quark confinement in nonabelian gauge theories, Phys. Rept. 23 (1976) 245; T. Suzuki, I. Yotsuyanagi, A possible evidence for abelian dominance in quark confinement, Phys. Rev. D 42 (1990) 4257.

[2] For reviews see T. Suzuki, Monopole condensation and confinement in SU(2) QCD, Nucl. Phys. Proc. Suppl. 30 (1993) 176; M. N. Chernodub, M. I. Polikarpov, Abelian projections and monopoles, in "Confinement, duality, and nonperturbative aspects of QCD", p. 387, ed. by P. van Baal, Plenum Press, 1997, [hep-th/9710205]; R. W. Haymaker, Confinement studies in lattice QCD, Phys. Rept. 315 (1999) 153 [hep-lat/9809094].

[3] V. Singh, D. A. Browne and R. W. Haymaker, Structure of Abrikosov vortices in SU(2) lattice gauge theory, Phys. Lett. B 306 (1993) 115 [hep-lat/9301004]; P. Cea and L. Cosmai, Dual superconductivity in the SU(2) pure gauge vacuum: A Lattice study, Phys. Rev. D 52 (1995) 5152 [hep-lat/ 9504008 ]; Y. Matsubara, S. Ejiri and T. Suzuki, The (dual) Meissner effect in SU(2) and SU(3) QCD, Nucl. Phys. Proc. Suppl. 34 (1994) 176, [hep-lat/9311061]; S. Kato et al., Various representations of infrared effective lattice SU(2) gluodynamics, [hep-lat / 9902013 ]; R. W. Haymaker and T. Matsuki, Consistent definitions of flux and the dual superconductivity parameters in SU(2) lattice gauge theory, [hep-lat/0505019].

[4] G. S. Bali, C. Schlichter and K. Schilling, Probing the QCD vacuum with static sources in maximal Abelian projection”, Prog. Theor. Phys. Suppl. 131 (1998) 645 [hep-lat/9802005].

[5] Y. Koma, M. Koma, E. M. Ilgenfritz and T. Suzuki, A detailed study of the Abelian-projected SU(2) flux tube and its dual Ginzburg-Landau analysis, Phys. Rev. D 68 (2003) 114504 [hep-lat/0308008].

[6] T. Suzuki, K. Ishiguro, Y. Mori, T. Sekido, The dual Meissner effect and Abelian magnetic displacement currents”, Phys. Rev. Lett. 94 (2005) 132001 [hep-lat/ 0410001 ]; The dual Meissner effect in SU(2) Landau gauge, [hep-lat/0410039].

[7] F. V. Gubarev, L. Stodolsky, V. I. Zakharov, On the significance of the quantity $A^{2}$, Phys. Rev. Lett. (2001) 862220 [hep-ph / 0010057$]$; F. V. Gubarev, V. I. Zakharov, On the Emerging Phenomenology of $\left\langle\left(A_{\mu}\right)^{2}\right\rangle$ Phys. Lett. (2001) 50128 [hep-ph/0010096].

[8] S. Maedan and T. Suzuki, An Infrared Effective Theory Of Quark Confinement Based On Monopole Condensation, Prog. Theor. Phys. 81 (1989) 229.

[9] M. N. Chernodub, Katsuya Ishiguro, Yoshihiro Mori, Yoshifumi Nakamura, M.I. Polikarpov, Toru Sekido, Tsuneo Suzuki, V. I. Zakharov, Vacuum type of SU(2) gluodynamics in maximally Abelian and Landau gauges, [hep-lat/0508004].

[10] Y. Iwasaki, Renormalization group analysis of lattice theories and improved lattice action: two-dimensional nonlinear O(n) sigma model, Nucl. Phys. B 258 (1985) 141.

[11] M. Albanese et al. [APE Collaboration], Glueball masses and string tension in lattice QCD, Phys. Lett. B 192 (1987) 163.

[12] S. Hioki et al., Gauge fixing ambiguity and monopole, Phys. Lett. B 271 (1991) 201.

[13] V. G. Bornyakov et al., Anatomy of the lattice magnetic monopoles, Phys. Lett. B 537 (2002) 291. 